\title{
Desenvolvimento de um modelo para avaliar a sustentabilidade corporativa
}

\author{
Fábio Cristiano Zamcopéa ${ }^{*}$, Leonardo Ensslin ${ }^{\mathrm{b}}$, Sandra Rolim Ensslin ${ }^{\mathrm{c}}$ \\ a"fabio@deps.ufsc.br, UFSC, Brasil \\ bleonardoensslin@gmail.com, UFSC, Brasil \\ 'sensslin@mbox1.br, UFSC, Brasil
}

\section{Resumo}

Este trabalho apresenta o desenvolvimento de um modelo de avaliação da sustentabilidade corporativa, construído com base no sistema de valores, interesses e preferências dos decisores de uma indústria têxtil. Como instrumento de intervenção utilizou-se a metodologia multicritério de apoio à decisão - construtivista (MCDA-C), por essa possibilitar a identificação de um conjunto de indicadores de desempenho que caracterizam a sustentabilidade e por mensurar o grau de alcance das propriedades analisadas da organização quanto ao seu compromisso com a sustentabilidade. 0 modelo possibilitou aos decisores a compreensão das principais questões de sustentabilidade e ações necessárias para resolvê-las, além de proporcionar a medição de desempenho e avaliação do progresso para melhorias contínuas. Entre as ações geradas a partir do modelo, destaca-se a criação de um comitê de sustentabilidade, revisão do planejamento estratégico, fortalecimento dos canais de comunicação e programas de conscientização para os funcionários, fornecedores e demais envolvidos.

\section{Palavras-chave}

Apoio à decisão. Avaliação de desempenho. MCDA-C. Sustentabilidade corporativa.

\section{Introdução}

Embora tenha emergido como uma ideia política na agenda internacional, o conceito de desenvolvimento sustentável tem sido cada vez mais aplicado às empresas nas últimas décadas (GLADWIN; KENNELY; KRAUSE, 1995; CALLENS; TYTECA, 1999; HANLEY, 2000; BELLEN, 2006; CAMPOS; MELLO; MEURER, 2007). Há quase 10 anos, já era comum aceitar o fato de que, sem o apoio das empresas, a sociedade nunca alcançaria o desenvolvimento sustentável devido às firmas representarem os recursos produtivos da economia (BANSAL, 2002). Para Azapagic e Perdan (2000), apesar de a indústria muitas vezes ser vista como fonte da degradação ambiental e da preocupação social, é amplamente reconhecido que a indústria é parte essencial para o desenvolvimento e criação de riqueza.

Nascimento, Lemos e Mello (2008) preconizam que é preciso mudar a forma de pensar e agir em relação às questões socioambientais, muito mais geradoras de oportunidades do que de custos, sendo possível conciliar sustentabilidade econômica com sustentabilidade social e ambiental. As empresas se deparam com o desafio de como traduzir os princípios gerais do desenvolvimento sustentável em prática dos negócios, e enfrentar esse problema requer uma abordagem sistêmica na qual a sustentabilidade corporativa não seja considerada um mero suplemento, mas seja sistematicamente integrada a todas as atividades empresariais.

Por outro lado, isso exige um modelo de gestão da sustentabilidade que permita: (i) compreensão das principais questões de sustentabilidade e ações necessárias para resolvê-las; (ii) medição de desempenho e avaliação do progresso para garantir melhorias contínuas; e (iii) comunicação das políticas e progresso de sustentabilidade para as partes interessadas (AZAPAGIC, 2003).

Diante do contexto evidenciado, o presente estudo tem o seu foco nas seguintes perguntas da 
pesquisa: (i) como pode ser identificado um conjunto de indicadores de desempenho que caracterizem a sustentabilidade da empresa? (ii) como pode ser mensurado o grau de alcance das propriedades analisadas da organização quanto ao seu compromisso com a sustentabilidade, tanto individual quanto globalmente?

Para responder essas questões, foi aplicada a metodologia multicritério de apoio à decisão - construtivista (MCDA-C) no estudo de caso, metodologia esta capaz de lidar com problemas complexos, adequada para a geração de conhecimento do contexto segundo os critérios julgados importantes pelos responsáveis no processo decisório e propor ações inovadoras com o objetivo de atingir o melhor nível de sustentabilidade empresarial.

0 objetivo principal deste trabalho é desenvolver um modelo de avaliação da sustentabilidade corporativa, construído com base no sistema de valores, interesses e preferências dos decisores de uma indústria têxtil.

Como objetivos específicos, cita-se:

- Efetuar a análise comparativa das abordagens e ferramentas de avaliação da sustentabilidade corporativa presentes na literatura;

- Identificar um conjunto de indicadores de desempenho que caracterizam a sustentabilidade corporativa; e

- Esclarecer os fatores relevantes que afetam os compromissos com a sustentabilidade, segundo a visão dos decisores nas diversas áreas da empresa.

Com a finalidade de atingir o objetivo proposto foi desenvolvido um estudo de caso, utilizando como instrumento de intervenção a metodologia multicritério de apoio à decisão - construtivista (MCDA-C) na construção de um sistema de avaliação da sustentabilidade corporativa, aplicado em uma indústria paranaense de médio porte. 0 método de procedimento da pesquisa (estudo de caso) foi escolhido por ser este um estudo de natureza empírica que investiga um determinado fenômeno, geralmente contemporâneo, dentro de um contexto real de vida, quando as fronteiras entre o fenômeno e o contexto em que ele se insere não são claramente definidas (BERTO; NAKANO, 2000).

0 motivo de desenvolver o modelo de avaliação com a metodologia MCDA-C é por esta se preocupar em: (i) extrair do decisor os aspectos considerados relevantes para fins de avaliação do desempenho, no contexto interno de seu subsistema e no contexto das inter-relações; (ii) mensurar esses aspectos, em termos de uma escala; e (iii) integrar todos esses aspectos, de forma a possibilitar uma visão global do sistema de avaliação (DUTRA, 2003).

Como limitação do estudo, pode-se dizer que, devido o estudo de caso tratar de um único caso, existe o risco de um julgamento incompleto em função de ser um evento único (SOUZA, 2005), visto que o modelo de avaliação foi construído para aplicação específica na empresa em que foi desenvolvido o trabalho, o que não impede a utilização do processo em outros contextos, desde que observadas as alterações pertinentes a outros negócios ou setores.

\section{Gestão e avaliação da sustentabilidade corporativa}

A sustentabilidade tornou-se uma das principais questões do século 21. Ela incorpora a promessa de evolução para uma sociedade mundial mais justa e próspera, em que o ambiente natural e as nossas realizações culturais sejam preservados para as próximas gerações (BELLEN, 2006; NASCIMENTO; LEMOS; MELLO, 2008; TRIGUEIRO, 2008).

0 termo gestão da sustentabilidade aborda os aspectos sociais, ambientais e econômicos da gestão empresarial em geral e em particular, da gestão da sustentabilidade corporativa (EPSTEIN; ROY, 2003; SCHALTEGGER; BURRITT; PETERSEN, 2003). A sustentabilidade corporativa reflete a meta final do movimento das empresas quanto à responsabilidade empresarial em cumprimento com os padrões de desempenho sustentável em relação às expectativas dos stakeholders ou grupos de interesse (BHIMANI; SOONAWALLA, 2005). 0 desempenho sustentável pode ser definido como a performance de uma empresa em todas as dimensões, que exige um modelo de gestão que estabeleça a ligação entre gestão ambiental e social com negócio e estratégia competitiva, além de integrar informação ambiental e social com informação econômica da empresa (KEEBLE; TOPIOL; BERKELEY, 2002; EPSTEIN; ROY, 2003).

A gestão do desempenho em sustentabilidade requer um modelo ligado à estratégia empresarial e que reporte ou faça a comunicação dessa sustentabilidade aos stakeholders (SCHALTEGGER; WAGNER, 2006). Na tentativa de facilitar a convergência e padronização de abordagens de gestão da sustentabilidade corporativa, Azapagic (2003) criou um modelo de sistema de gestão, potencialmente aplicável a qualquer indústria, concebido para servir como guia para as organizações na definição de uma estratégia de sustentabilidade e de um plano de ação para tornar as empresas mais sustentáveis.

A avaliação do desempenho em sustentabilidade pode ser definida como a medição e gestão da interação entre empresas, sociedade e meio ambiente (BENNETT; JAMES, 1997). Os problemas e perspectivas da avaliação do desempenho em sustentabilidade corporativa podem ser analisados em três níveis: 0 nível individual dos indicadores de desempenho, o 
nível global do sistema de avaliação de desempenho e o nível da relação deste com o ambiente externo (NEELY, 1993; BELL; MORSE, 1999).

Os interesses de vários stakeholders conduzem o desenvolvimento da avaliação de desempenho da sustentabilidade (WEHRMEYER; TYTECA, 1998). Schaltegger e Wagner (2006) afirmam que a avaliação do desempenho da sustentabilidade requer dos gestores definição de metas e critérios que sejam entendidos pela empresa, comunicação e interação com os stakeholders e que estabeleçam informação, avaliação e um sistema de relatório que dê suporte à gestão e comunicação.

Keeble, Topiol e Berkeley (2002) argumentam que o desenvolvimento de indicadores de sustentabilidade nas empresas não é tarefa fácil, além de exigir um alto envolvimento das pessoas que serão responsáveis pelos apontamentos desses indicadores. Muitas vezes as organizações investem recursos significativos no sentido de encontrar o conjunto "ideal" de indicadores, quando, na verdade, o desenvolvimento e a utilização de indicadores deveria ser um processo dinâmico, com o objetivo de auxiliar na tomada das decisões.

\section{Indicadores de sustentabilidade corporativa}

Na pesquisa das abordagens de mensuração da sustentabilidade, com foco nas três dimensões clássicas (econômica, social e ambiental) da sustentabilidade e no envolvimento com os stakeholders, foram selecionadas as mais utilizadas internacionalmente no meio empresarial (LOUETTE, 2007; CAMPOS; LEMME, 2007) e, adicionalmente, duas iniciativas brasileiras, que em sua construção levou em consideração os critérios apresentados. Essas ferramentas de sustentabilidade corporativa são:

- Dow Jones Sustainability Indexes - DJSI;

- FTSE4Good Index;

- Global Reporting Initiative - GRI;

- Índice de Sustentabilidade Empresarial - ISE; e

- Indicadores Ethos de Responsabilidade Social.

0 Índice Dow Jones de Sustentabilidade (Dow Jones Sustainability Index - DJSI) foi lançado em 1999 pela Dow Jones Indexes em conjunto com a Sustainable Asset Management (D0W..., 2008). 0 indice mede o desempenho financeiro e fatores econômicos, além do envolvimento social, cultural e ambiental das empresas.

A seleção das empresas é feita a partir de um amplo questionário centrado no desempenho ambiental, social e econômico, incluindo indicadores de governança corporativa, gerenciamento de risco, gestão da marca, padrões da cadeia de suprimentos e práticas trabalhistas (LOUETTE, 2007). Para as companhias, além do efeito benéfico sobre a imagem institucional, o ingresso no índice também pode ter um impacto positivo sobre as ações negociadas em Bolsa porque abre um mercado potencial superior a US\$ 4 trilhões, oriundos de fundos direcionados a empresas social e ambientalmente responsáveis, segundo dados da ONU.

Para elaborar o ranking das empresas é feita uma avaliação do grau de sustentabilidade por meio da análise de três dimensões (Tabela 1):

0 DJSI adota a metodologia conhecida como Best in Class, que seleciona as empresas com melhor desempenho em cada um dos setores econômicos, excluindo apenas organizações do setor de defesa com mais de $50 \%$ do faturamento proveniente da venda de armas (MAZON; MONZONI; RIBEIRO, 2005).

Com base na experiência americana, a Bolsa de Londres e o Financial Times lançaram em 2001 o FTSE4Good Index. A série é composta por quatro índices e foi desenvolvida pela empresa de pesquisa EIRIS para avaliar o desempenho de empresas globais por meio de critérios ambientais, de direitos humanos e de engajamento de stakeholders (MAZON; MONZONI; RIBEIRO, 2005).

Os critérios são avaliados segundo três aspectos: políticas (comitês de monitoramento, metas, código de conduta), gestão (acidentes públicos, planos de assistência, seguros) e reporting (estatísticas e relatórios ambientais). A metodologia utilizada é o screening negativo, sendo excluídas as indústrias do tipo bélica, nuclear e tabagista. Os critérios são constantemente aprimorados para assegurar que reflitam padrões de responsabilidade corporativa e estejam alinhados com o conceito de investimento socialmente responsável (FINANCIAL..., 2005).

0 modelo GRI foi criado pela Global Reporting Initiative (GRI), organização internacional com sede em Amsterdã, que tem como missão desenvolver

Tabela 1. Critérios de avaliação da sustentabilidade corporativa.

\begin{tabular}{clc}
\hline Dimensão & \multicolumn{1}{c}{ Critério } & Ponderação \\
\hline \multirow{2}{*}{ Econômica } & Governança corporativa & 6,0 \\
& Gestão de risco e crise & 6,0 \\
& Código de conduta & 6,0 \\
& Critério específico da indústria & - \\
\hline \multirow{2}{*}{ Ambiental } & Relatório ambiental & 3,0 \\
& Critério específico da indústria & - \\
\hline \multirow{4}{*}{ Social } & Práticas trabalhistas & 5,0 \\
& Desenvolvimento de capital & 5,5 \\
& humano & 5,5 \\
& Atração e retenção de talentos & 3,0 \\
& Cidadania corporativa & 3,0 \\
& Relatório social & - \\
\hline
\end{tabular}

Fonte: Dow Jones Sustainability Index, 2009. 
e disseminar globalmente diretrizes e incorporar indicadores de sustentabilidade para a elaboração de relatórios (Sustainability Reporting Guidelines). Ele determina princípios e estrutura para o relatório, o que permite às organizações a apresentação geral de seu desempenho econômico, social e ambiental, permitindo a comparação com o mercado, já que estabelece padrões (indicadores) e serve como plataforma para facilitar o diálogo e o engajamento de stakeholders (GLOBAL..., 2006).

Os princípios para a elaboração de relatórios de sustentabilidade são: materialidade, inclusão dos stakeholders, contexto da sustentabilidade, abrangência, equilíbrio, comparabilidade, exatidão, periodicidade, clareza e confiabilidade.

Outra ferramenta, o Índice Bovespa de Sustentabilidade Empresarial (ISE), foi apresentado em dezembro de 2005. Não há exclusão sumária de setores econômicos no índice, adotando o modelo screening positivo, onde todas as empresas com liquidez mínima no mercado definida pelo Conselho do ISE tem a oportunidade de preencher o questionário e participar da seleção (CAMPOS; LEMME, 2007).

0 objetivo principal do índice é criar um ambiente de investimento compatível com as demandas do desenvolvimento sustentável e estimular a responsabilidade ética corporativa (MAZON; MONZONI; RIBEIRO, 2005). 0 índice também se constitui como ferramenta para análise comparativa da performance das empresas listadas na Bovespa sob o aspecto da sustentabilidade corporativa.

Os critérios e indicadores foram definidos com base em princípios consagrados na literatura de sustentabilidade corporativa, como: (i) informação ao público; (ii) comparabilidade; (iii) auditabilidade/rastreabilidade; (iv) abrangência; e (v) temporalidade. 0 conceito base do ISE é o Triple Bottom Line (TBL), criado pela empresa de consultoria inglesa SustainAbility, que avalia elementos econômico-financeiros, sociais e ambientais de forma integrada (BOVESPA, 2005). Aos princípios do TBL foram acrescidos mais três grupos de indicadores: (i) critérios gerais (posição da empresa perante acordos globais e balanço social); (ii) critérios de natureza do produto; e (iii) critérios de governança corporativa.

Já os Indicadores Ethos de Responsabilidade Social Empresarial (RSE) servem como instrumento de autodiagnóstico, tendo como finalidade principal auxiliar as empresas a gerenciarem os impactos sociais e ambientais decorrentes de suas atividades. Foram criados pelo Instituto Ethos de Empresas e Responsabilidade Social, organização não governamental criada em 1998 por empresários e executivos do setor privado (LOUETTE, 2007), e têm por objetivo aferir o grau de sustentabilidade corporativa praticada na organização, a fim de obter melhor posicionamento estratégico da empresa, promovendo a longevidade em harmonia com a sustentabilidade dos mercados, sociedade e recursos naturais envolvidos.

A estrutura dos indicadores possibilita a empresa planejar o modo de alcançar um grau mais elevado de responsabilidade social, fornecendo parâmetros para os passos subsequentes e apontando diretrizes para o estabelecimento de metas de aprimoramento dentro do universo de cada tema (INSTITUTO..., 2008). 0 questionário contém questões binárias e questões quantitativas, sendo organizado em sete grandes temas (Quadro 1):

\section{Metodologia multicritério de apoio à decisão - construtivista}

A metodologia multicritério de apoio à decisão - construtivista (MCDA-C) consolidou-se como instrumento científico de gestão a partir da década de 1980 com os trabalhos de Roy (1985), Landry (1995), Bana e Costa (1992), dentre outros. A MCDA-C surgiu como uma ramificação da metodologia multicritério em apoio à decisão - MCDA, direcionada a apoiar os decisores em contextos complexos, conflituosos, incertos, onde estes desejavam melhorar seu entendimento da situação. Com o intuito de cumprir o objetivo ao qual se propõe, a MCDA-C utiliza-se de atividades de apoio à decisão, as quais são subdivididas em três fases: estruturação, avaliação e elaboração das recomendações (ENSSLIN; DUTRA; ENSSLIN, 2000).

Bana e Costa (1993) afirma que para o apoio à decisão, os atores e seus valores, objetivos e normas, somados às ações e suas características, são elementos que estruturam o contexto decisório. Esse conjunto de atividades, que tem por objetivo gerar entendimento aos decisores com base em seu sistema de valores, é

Quadro 1. Temas e subtemas dos Indicadores Ethos.

\begin{tabular}{|c|l|}
\hline \multicolumn{1}{|c|}{ Tema } & \multicolumn{1}{c|}{ Subtema } \\
\hline $\begin{array}{c}\text { Valores, transparência e } \\
\text { governança }\end{array}$ & $\begin{array}{l}\text { Autorregulação da conduta; } \\
\text { relações transparentes com a sociedade }\end{array}$ \\
\hline Público interno & $\begin{array}{l}\text { Diálogo e participação; respeito ao } \\
\text { indivíduo; } \\
\text { trabalho decente }\end{array}$ \\
\hline Meio ambiente & $\begin{array}{l}\text { Responsabilidade com as gerações } \\
\text { futuras; } \\
\text { gerenciamento do impacto ambiental }\end{array}$ \\
\hline Fornecedores & Seleção, avaliação e parceria \\
\hline Consumidores e clientes & Dimensão social do consumo \\
\hline Comunidade & $\begin{array}{l}\text { Relações com a comunidade local; } \\
\text { ação social }\end{array}$ \\
\hline Governo e sociedade & $\begin{array}{l}\text { Transparência política; } \\
\text { liderança social }\end{array}$ \\
\hline
\end{tabular}

Fonte: Instituto Ethos de Responsabilidade Social, 2009. 
chamado estruturação. A estruturação do contexto decisório é que vai capacitar o decisor a melhor alcançar amplo entendimento ao tratar dos problemas (MILLER, 1970). A estruturação se constitui na fase cuja elaboração é o fator mais crítico ao sucesso no apoio à decisão, pois visa entender o problema e todo o contexto no qual está inserido (DUTRA, 2003). Nessa primeira fase são construídos os mapas cognitivos dos decisores. Em seguida, é efetuada a análise do mapa cognitivo a partir da identificação das características estruturais e do conteúdo do mapa e, por fim, é determinada a família de pontos de vista fundamentais (PVFs) que correspondem às dimensões que o decisor julga necessárias e suficientes para avaliar o contexto.

A segunda fase consiste no modelo de avaliação das alternativas, que é composto pela construção do modelo em si, por meio da construção da escala de preferência local de cada PVF e da determinação das taxas de compensação ou substituição, bem como a identificação do perfil de impacto das ações e avaliação global. A fase de avaliação consiste na elaboração de um processo de determinar as preferências entre as consequências, realizado pela comparação entre as alternativas de acordo com índices de valor (SIMON, 1979). Comparar duas ações em termos de preferência é a saída quando se depara com aspectos conflitantes que se encontram na mente dos diferentes atores (ROY, 1994). E isso se dá por uma abordagem interativa, iterativa, recursiva e construtiva do aprendizado. Como a avaliação é limitada em sua exatidão de poder acompanhar os vários elementos de valor na consequência imaginada, a experiência prévia permite que se façam inferências (SIMON, 1979).

$\mathrm{Na}$ terceira fase, ou fase de recomendações, é desenvolvido o processo de gerar ações para o contexto, a análise e a avaliação das performances de cada aspecto julgado relevante, o que canaliza as discussões entre os envolvidos para o que é mais importante dentro do contexto decisório (ENSSLIN; CAMPOS, 2006). As ações geradas podem ser organizadas em estratégias para aperfeiçoamento e aprimoramento da performance que melhor atendam aos objetivos dos decisores. Bana e Costa et al. (1999) afirmam que recursividade no processo de construção do modelo torna o MCDA-C mais versátil e flexível, ao permitir o feedback aos atores em qualquer estágio do processo, o que concede aos atores o poder de reverem seus conceitos em qualquer momento que desejem, à medida que forem obtendo maior conhecimento do problema.

Ao empregar a visão construtivista, a metodologia MCDA-C foca sua ação na estruturação do contexto, constrói entendimento da situação e dos objetivos envolvidos, como uma forma de mensurá-los. Devido ao processo empregado pelo MCDA-C ser interativo, permitindo a realimentação por parte dos atores em qualquer estágio do processo (revisão de conceitos à medida que os atores obtêm maior conhecimento do problema), requer a participação e legitimação de todas as etapas pelo decisor, exigindo dedicação e esforço (ENSSLIN, 2002). Se não ocorrer a constante legitimação da construção do modelo por parte do decisor, ele poderá perder o interesse no processo, por considerar que o modelo em construção não mais lhe pertence.

\section{Metodologia científica}

Neste estudo, utilizou-se a pesquisa exploratória de abordagem qualitativa e o método de procedimento foi o estudo de caso. Segundo Godoy (1995), uma pesquisa qualitativa deve ter como preocupação maior a interpretação de fenômenos e a atribuição de resultados. 0 estudo de caso é um estudo de natureza empírica que investiga um determinado fenômeno, geralmente contemporâneo, dentro de um contexto real de vida, quando as fronteiras entre o fenômeno e o contexto em que ele se insere não são claramente definidas. Trata-se de uma análise aprofundada de um objeto (caso), que permita o seu amplo e detalhado conhecimento (BERTO; NAKANO, 2000). Seu objetivo é aprofundar o conhecimento acerca de um problema não suficientemente definido, visando estimular a compreensão, sugerir hipóteses e questões ou desenvolver a teoria (MATTAR, 1996).

A sequência para a condução do estudo de caso foi baseada nos trabalhos de Forza (2002), Croom (2005), Souza (2005) e Miguel (2007), sendo compreendida pelas seguintes fases: (i) definição da estrutura conceitual-teórica; (ii) planejamento do caso; (iii) coleta dos dados; (iv) análise dos dados; e (v) geração de relatório. No presente estudo, a fase de geração de relatório compreende toda a estruturação do modelo e suas recomendações.

A definição da estrutura conceitual-teórica foi alcançada por meio do mapeamento da literatura de sustentabilidade corporativa. As bases de dados pesquisadas foram Web of Science, Science Direct, Wilson Web, Blackwell, Econlit e Proquest, além de pesquisas em livros e sites direcionados a pesquisas acadêmicas (dissertações, teses, artigos).

No planejamento do caso, para maior aprofundamento na investigação, determinou-se o estudo de um único caso. 0 estudo de caso apresenta como unidade de análise uma indústria de médio porte do ramo têxtil, com mais de 500 funcionários, localizada no estado do Paraná, presente nas principais lojas da linha homewear no Brasil.

A escolha da empresa ocorreu devido à necessidade informada pelos diretores da empresa, de refletirem a 
estratégia de condução dos negócios, reconhecendo que a atividade empresarial necessita gerar valor além do resultado financeiro e alcançar o patamar de agente de transformação socioambiental.

A partir da seleção do caso, foram determinados os métodos e técnicas para a coleta e para a análise dos dados. Foram empregadas múltiplas fontes de evidência, através de entrevista aberta, realizada com os diretores da empresa (diretor de operações, diretor financeiro e diretora de marketing e criação), gerente de $\mathrm{RH}$, gerente de compras e gerente de vendas. A utilização de diversas fontes de evidência permite a iteração entre as diversas fontes de evidência para sustentar os construtos, proposições ou hipóteses, visando analisar a convergência das fontes de evidência (MIGUEL, 2007).

Para a coleta de dados secundários, assim como para o levantamento de informações relevantes à investigação, utilizaram-se técnicas de documentação direta e indireta, e observação direta intensiva (COOPER; SCHINDLER, 2003). Foram realizadas diversas visitas à empresa para construção do modelo, sendo constantes as interações com os decisores (fontes de evidência) para a validação deste.

$\mathrm{Na}$ fase de estruturação da metodologia do instrumento utilizado (MCDA-C), foram criados os mapas cognitivos no Powerpoint e na fase de avaliação foi empregado o software Macbeth (BANA e COSTA; DE CORTE; VANSNICK; Versão 1.1, 2005), desenvolvido em 1995 (BANA e COSTA; VANSNICK, 1995), para a construção das escalas e do cálculo das taxas de substituição. 0 Excel foi utilizado nas matrizes de julgamento (ROBERTS, 1979) e no traçado do perfil de impacto. Para a realização da análise de sensibilidade, foi utilizado o programa Hiview (KRYSALIS; versão 2.00B, 1995), criado por Barclay em 1984 (BARCLAY, 1984).

\section{Estudo de caso}

Esta seção apresenta a construção do modelo de avaliação da sustentabilidade corporativa, baseado no sistema de valores, interesses e preferências dos decisores, levando em consideração os requisitos dos stakeholders (público de interesse) na percepção desses decisores. Trata-se de uma empresa de médio porte do ramo têxtil, localizada no estado do Paraná, que emprega mais de 500 colaboradores diretos, com presença nas principais lojas da linha homewear no Brasil e em outros países. 0 modelo foi construído com base na metodologia multicritério de apoio à decisão - construtivista (MCDA-C).

\subsection{Identificação do contexto decisório}

0 desenvolvimento da pesquisa teve início ao deparar-se com a necessidade apresentada pela diretoria da empresa, preocupada em avaliar e direcionar a sua gestão visando a sustentabilidade. A diretoria visualizou a necessidade de fundamentar suas decisões estratégicas e táticas, assessoradas por uma ferramenta capaz de avaliar, monitorar e aperfeiçoar as ações e práticas da organização e de sua cadeia, em busca da criação de valor a longo prazo.

Nessa primeira etapa de construção do modelo, foi caracterizado o processo decisório, com os atores envolvidos, as ações a serem avaliadas e as diversas formas possiveis de avaliação das ações (ENSSLIN; DUTRA; ENSSLIN, 2000). No processo decisório, foram definidos como atores: diretor de Operações, diretor Financeiro, diretora de Marketing e Criação, gerente de RH, gerente de Compras e gerente de Vendas - buscando dessa forma representar os interesses dos principais stakeholders da empresa (funcionários, sócios, fornecedores, parceiros, clientes).

Tendo em vista o número de atores diretamente envolvidos ser elevado, estabeleceu-se o critério do consenso nas questões de opiniões contraditórias. Os decisores que ao final selecionavam o que pertence ao problema e o que não pertence foram o diretor de Operações e a diretora de Marketing. Como facilitador, configurou-se o autor do presente trabalho, assumindo a função de apoiar o processo de tomada de decisão através da construção do modelo; como agidos, foram identificados os consumidores dos produtos da empresa, a comunidade local, o governo (federal, estadual e municipal) e a sociedade em geral.

A problemática levantada pelos decisores fundamenta-se na preocupação com o valor da empresa a longo prazo e as consequências de suas ações perante a sociedade e meio ambiente. Buscar a sustentabilidade não para mostrar-se ao mercado, mas para conseguir a conscientização e consentimento dos stakeholders para orientar as políticas de longo prazo. Sendo assim, a problemática é a de como avaliar a gestão da sustentabilidade corporativa, buscando constantemente melhorar a performance das ações, com vistas a sua longevidade e a uma contribuição mais efetiva à sociedade e ao meio ambiente.

\subsection{Estruturação do problema}

Nessa etapa, o primeiro passo foi definir junto aos decisores um rótulo que descrevesse o problema. 0 rótulo foi definido como "desenvolver um modelo para avaliar o desempenho da sustentabilidade corporativa". Com base nesse rótulo, partiu-se para a definição dos elementos primários de avaliação (EPA) por meio de um brainstorming (OSBORN, 1993) com os decisores.

Os EPAs são as primeiras preocupações manifestadas pelos decisores, quando estes confrontam a situação (KEENEY, 1992) e, segundo Schaefer (2005), esses elementos são constituídos de objetivos, metas, 
valores do decisor, ações, opções e alternativas que servirão de base para a construção do mapa cognitivo.

Foram levantados 77 EPAs junto aos decisores e, na sequência, esses EPAs foram orientados à ação e transformados em conceitos (Quadro 2), onde foram identificados os polos presentes (pretendido) e os polos opostos psicológicos (mínimo aceitável pelo decisor). Sendo assim, o mapa, através de seus polos presentes, pode fornecer uma indicação de personalidade, atitudes e proposições gerais dos decisores, bem como os aspectos culturais da organização (EDEN; JONES; SIMS, 1983).

0 passo seguinte foi organizar, juntamente com os decisores, os EPAs e conceitos em grandes áreas. As grandes áreas para a avaliação da sustentabilidade foram definidas conforme o tripé da sustentabilidade ou Triple Bottom Line (ELKINGTON, 1997; DYLLICK; HOCKERTS, 2002), sendo as dimensões econômica, social e ambiental, conforme Figura 1.

A partir dos conceitos organizados, partiu-se para a construção dos mapas cognitivos, sendo um mapa cognitivo para cada área. Com os mapas cognitivos prontos, foi realizada a análise avançada destes, identificando os clusters e, em cada cluster, os ramos de acordo com as linhas de argumentação de cada mapa.

Esses ramos são constituídos por uma ou mais linhas de argumentação que demonstrem preocupações similares sobre o contexto decisório (ENSSLIN; MONTIBELLER FILHO, 1998). É essencialmente uma análise de conteúdo que leva em conta as ideias expressas nos conceitos. Sobre cada um dos ramos do mapa foi feita a pesquisa visando identificar os pontos de vista que os decisores desejavam levar em conta no modelo multicritério.

\subsection{Estruturação do modelo multicritério}

0 modelo multicritério utiliza-se de uma estrutura arborescente de valor (BELTON, 1990) e a transição de um mapa cognitivo para um modelo multicritério acontece através da identificação das dimensões

Quadro 2. Os quatro primeiros elementos primários de avaliação e seus respectivos conceitos.

\begin{tabular}{|l|l|}
\hline \multicolumn{1}{|c|}{ EPA } & \multicolumn{1}{c|}{ Conceito } \\
\hline 1. Princípios éticos & $\begin{array}{l}\text { C1. Estabelecer princípios éticos para guiar a conduta... ter desvios de conduta e não } \\
\text { poder justificar as penalidades }\end{array}$ \\
\hline 2. Compromisso com o desenvolvimento sustentável & $\begin{array}{l}\text { C2. Ter compromisso com o desenvolvimento sustentável... não ter comprometimento } \\
\text { com a vida e com futuras gerações }\end{array}$ \\
\hline 3. Sensibilização de funcionários e fornecedores & $\begin{array}{l}\text { C3. Efetuar programas de sensibilização de funcionários e fornecedores... não } \\
\text { influenciar e despertar o interesse pelo tema de sustentabilidade }\end{array}$ \\
\hline 4. Transparência & $\begin{array}{l}\text { C4. Ser transparente em suas ações... não alcançar a confiança de seus clientes, } \\
\text { funcionários, fornecedores e comunidade }\end{array}$ \\
\hline . & $\begin{array}{l}\text {. } \\
\text {. }\end{array}$ \\
\hline
\end{tabular}

Fonte: dados da pesquisa.

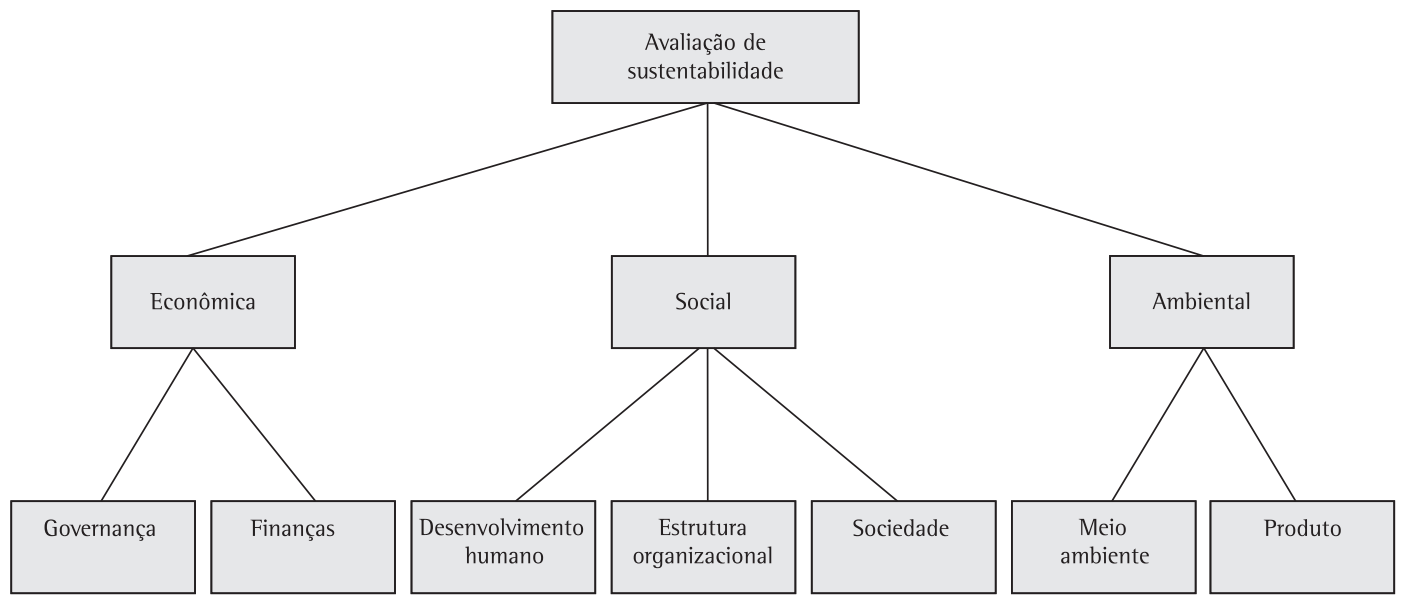

$01,02,03,04,05, \quad 09,28,29,30,31,10,45,46,47,48,12,14,19,34,50, \quad 17,43,44,60,61,22,23,24,35,37,11,13,16,25,26$ 06, 07, 08, 15, 18, $\quad 32,33 \quad 49,53,54,57,58,51,52,55,56,72, \quad 62,63,64,65,74 \quad 38,39,40,41,42 \quad 27,66,67,68,69$, $20,21,36,77$ 59,75 $70,71,73$

Figura 1. Áreas e subáreas do modelo de avaliação de sustentabilidade. Fonte: dados da pesquisa. 
identificadas pelos decisores como necessárias e suficientes para representar o problema. A esse conjunto de dimensões denomina-se família de pontos de vista fundamentais (PVFs).

Os PVFs explicitam os valores que os decisores consideram importante naquele contexto, definindo ao mesmo tempo as características das ações que são de interesse dos decisores (GIFFHORN, 2007).

A partir dos PVFs e, de acordo com a decomposição, identificando os pontos de vista elementares (PVEs), seguiu-se para a criação dos descritores. De acordo com Ensslin, Montibeller e Noronha (2001), um descritor é definido como um conjunto de níveis de impacto que servem como base para avaliar a performance das ações potenciais em termos de cada ponto de vista - PV. Cada nível de impacto representará o desempenho de uma ação potencial nesse objetivo. Foram construídos 99 descritores, como o descritor comprometimento exemplificado na Figura 2.

Os descritores foram construídos e foram definidos para cada um deles, dois níveis de impacto de referência, sendo o nível superior chamado bom (100) e o nível inferior chamado neutro (0). Os níveis de 0 a 100 estão em compatibilidade com 0 mercado. Acima de 100 é considerado desempenho de excelência e abaixo de 0 a empresa está com desempenho abaixo do mercado. Esses níveis são importantes também para verificar a independência preferencial e também para determinar as taxas de substituição (BANA E COSTA et al., 1999). Para melhor visualização da performance do estado atual (statu quo) foi traçado um perfil de impacto nos eixos de avaliação do modelo, onde graficamente puderam ser representados no eixo horizontal os 99 critérios e no eixo vertical o desempenho atual em cada critério.
Após a definição dos descritores, foi realizado o julgamento de quanto a performance de cada ação é atrativa, definindo assim uma função de valor associada ao descritor, ou seja, transformando o descritor (escala ordinal) em um critério de mensuração (escala de intervalo) segundo o sistema de valores dos decisores. 0 método utilizado para a construção das funções de valor foi o método do julgamento semântico, onde a função de valor é obtida através de comparações par a par da diferença de atratividade entre as ações potenciais (BEINAT, 1995).

As comparações foram feitas conforme o que os decisores expressaram qualitativamente, por meio de uma escala ordinal semântica, a intensidade de preferência de uma ação sobre a outra. Para isso, foi utilizado o software Macbeth (Measuring Attractiveness by a Categorial Based Evaluation Technique) desenvolvido por Bana e Costa, de Corte e Vansnick, em 1995. Os critérios de mensuração permitiram avaliar a performance local das ações.

Para determinar a performance global de cada ação, partiu-se para a determinação das taxas de substituição dos critérios. As taxas de substituição de um modelo multicritério de avaliação expressam a perda de performance que uma ação potencial sofrerá em um critério para compensar o ganho de desempenho em outro (KEENEY; RAIFFA, 1993; ROY, 1996). Para determinar as taxas de substituição foi utilizado o método da comparação par a par, onde foi ordenada a preferência dos critérios com o auxílio de matriz de ordenação (ROBERTS, 1979). Em seguida, utilizou-se o software Macbeth para, através de julgamentos semânticos, calcular as taxas de substituição que melhor representavam numericamente os julgamentos.

\subsection{Comprometimento}

Com relação ao comprometimento dos sócios e da diretoria nas questões de sustentabilidade do negócio

Nível bom 1 Nivel neutro

Figura 2. Descritor comprometimento e seus níveis de impacto. Fonte: dados da pesquisa 


\subsection{Avaliação das ações potenciais}

Com o modelo de avaliação estruturado, o passo seguinte foi utilizá-lo para avaliar a performance das ações e gerar alternativas junto aos decisores. Para tanto, utilizou-se do Hiview (BARCLAY, 1984), que é um software para a análise de sensibilidade, que pode ser usado em processos de apoio à decisão que utilizam uma função de agregação aditiva (ENSSLIN; NORONHA, 1997). Esse aplicativo confronta situações em que existem múltiplas escolhas e em que se pretende selecionar a melhor opção.

Uma vez que os pontos de vista foram construídos e testados quanto à independência preferencial ordinal e cardinal, pode-se representar a performance global por meio do modelo de agregação aditiva (modelo compensatório), conforme proposto pela Equação 1:

$v(a)=w_{1}^{*} v_{1}(a)+w_{2}^{*} v_{2}(a)+$

$w_{3}{ }^{*} v_{3}(a)+\ldots+w_{n}^{*} v_{n}(a)$

onde,

$\mathrm{V}(\mathrm{a})=$ valor global da ação potencial "a"

$\mathrm{V}_{1}(\mathrm{a}), \mathrm{V}_{2}(\mathrm{a}), \mathrm{V}_{3}(\mathrm{a}), \ldots, \mathrm{V}_{\mathrm{n}}(\mathrm{a})=$ valor parcial dos critérios $1,2,3, \ldots, n$, da ação potencial "a"

$\mathrm{W}_{1}, \mathrm{~W}_{2}, \mathrm{~W}_{3}, \ldots, \mathrm{W}_{\mathrm{n}}=$ taxas de substituição dos critérios $1,2,3, \ldots, n$

$\mathrm{n}$ = número de critérios utilizados no modelo

A Equação 1 fornece a soma ponderada dos valores parciais obtidos por uma determinada ação "a" nos diversos critérios, sendo a ponderação feita pelas taxas de substituição de cada critério. Ao utilizar essa fórmula para calcular a situação atual (statu quo) chegou-se a um total de $-35,89$ pontos, demonstrando desempenho abaixo do nível de mercado (0 a 100).

\subsection{Análise dos resultados do modelo e recomendações}

A análise de sensibilidade permite que se conheça a variação que uma alteração na taxa de compensação de um critério ou na performance de uma ação poderá causar na avaliação das ações potenciais. 0 gráfico da Figura 3, gerado pelo software Hiview, mostra os 11 critérios de maior impacto do modelo, representando $29 \%$ do impacto total. Ou seja, obter um nível a mais na escala desses critérios causará um impacto positivo de 29\% na pontuação global. A Tabela 2 aumenta a compreensão, com a descrição completa dos critérios e seus PVFs (de onde foram ramificados). No entanto, nota-se uma pequena variação entre cada critério, visto que para completar a pontuação de 100\% existem mais 88 critérios.

Os cinco critérios de maior impacto representam $18,56 \%$ do impacto total. Ao obter-se um nível a mais na escala desses critérios, a pontuação total de -36 seria reduzida para -16 . Isso demonstra que é necessário conduzir ações de forma a trabalhar em paralelo vários critérios para entrar na posição de mercado (0 a 100) em um espaço menor de tempo.

A pontuação de $-35,89$ foi entendida pelos decisores como consequência da empresa nunca ter direcionado esforços para a questão da sustentabilidade, principalmente na área ambiental e social. 0 modelo foi de grande relevância por possibilitar aos decisores visualizarem a situação global da empresa, suas ações e respectivas consequências.

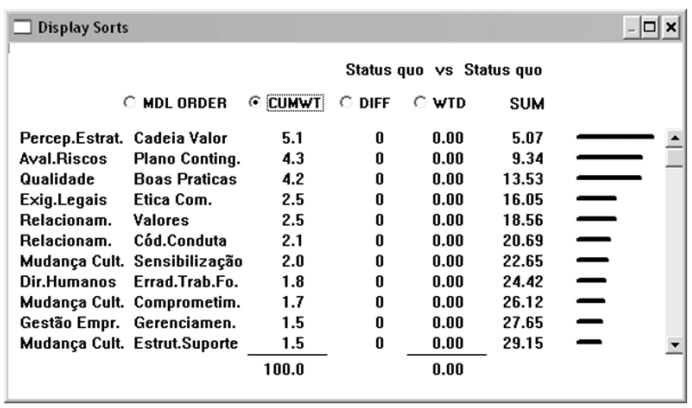

Figura 3. Os 11 critérios de maior impacto conforme taxa de substituição. Fonte: dados da pesquisa.

Tabela 2. Legenda da Figura 3 (11 critérios de maior impacto).

\begin{tabular}{llcc}
\hline \multicolumn{1}{c}{ PVF } & \multicolumn{1}{c}{ Critério (ramificação do PVF) } & Impacto global & Impacto global acumulado \\
\hline Percepção estratégica & Cadeia de valor & 5,1 & 5,07 \\
Avaliação de riscos & Plano de contingência & 5,3 & 9,34 \\
Qualidade & Boas práticas & 4,2 & 13,53 \\
Exigências legais & Ética comercial & 2,5 & 16,05 \\
Relacionamentos & Valores & 2,5 & 18,56 \\
Relacionamentos & Código de conduta & 2,1 & 20,69 \\
Mudança cultural & Sensibilização & 2,0 & 22,65 \\
Direitos humanos & Erradicação trabalho forçado & 1,8 & 24,42 \\
Mudança cultural & Comprometimento & 1,7 & 26,12 \\
Gestão empresarial & Gerenciamento & 1,5 & 27,65 \\
Mudança cultural & Estrutura de suporte & 1,5 & 29,15 \\
\hline
\end{tabular}

Fonte: dados da pesquisa. 


\subsection{Recomendações}

0 modelo construído teve como propósito aumentar o entendimento dos decisores sobre a sustentabilidade corporativa. Essa maior compreensão permitiu aos decisores identificarem, organizarem e mensurarem os aspectos críticos, bem como visualizarem o perfil da situação atual por meio dos indicadores. As recomendações a partir do modelo proposto foram sintetizadas em várias iniciativas, que no planejamento estratégico da empresa serão desdobradas em planos de ação e projetos. As principais iniciativas são:

- Criar comitê de sustentabilidade e dar treinamento ao comitê;

- Rever planejamento estratégico, considerando as questões de sustentabilidade;

- Estabelecer indicadores para acompanhar o alcance dos objetivos;

- Elaborar código de conduta e treinar funcionários sobre os conceitos;

- Realizar programas de sensibilização dos funcionários na questão da sustentabilidade;

- Mapear competências funcionais, gerenciais e organizacionais, bem como os níveis de decisão e responsabilidade;

- Desenvolver os gestores nas competências funcionais, gerenciais e organizacionais;

- Criar e treinar comitê de auditoria interna;

- Realizar avaliação de desempenho dos resultados da empresa mensalmente;

- Efetuar planejamento tributário juntamente com especialistas;

- Avaliar sistema de informações e dar treinamento para o pessoal que opera o sistema;

- Elaborar plano de contingência e testar plano;

- Efetuar avaliação de desempenho dos funcionários;

- Avaliar necessidades e programar anualmente treinamento interno e externo dos funcionários;

- Efetuar pesquisa salarial a cada dois anos e analisar amplitude dos níveis salariais;

- Criar a participação por resultados;

- Criar canal especializado no RH para ouvir empregados;

- Elaborar normas de proibição de práticas discriminatórias e conscientizar funcionários;

- Elaborar normas de proibição de práticas de assédio moral e sexual e conscientizar funcionários;

- Criar canal confidencial para avaliar processo de contratação sem discriminação;

- Manter vagas para portadores de deficiência e reabilitados;
- Manter vagas para menor aprendiz;

- Manter campanhas regulares de conscientização sobre saúde e segurança;

- Fornecer informações sobre a empresa aos funcionários e divulgar organograma;

- Realizar treinamentos sobre novos produtos e novas políticas;

- Elaborar manual de boas práticas de fabricação;

- Criar programa de incentivo a sugestões;

- Realizar atividades sistemáticas de integração dos funcionários;

- Mapear cargos estratégicos e criar planos de sucessão;

- Manter relação contratual, monitorar requisitos com trabalhadores terceirizados e repassar alguns benefícios;

- Reunir com representantes locais, assistentes sociais e ONGs para levantar necessidades para projetos sociais;

- Incentivar o trabalho voluntário e divulgar projetos sociais entre funcionários;

- Atuar na melhoria de espaços públicos;

- Elaborar projetos em parceria com o poder público para melhorias de escolas/creches;

- Monitorar fornecedores no tratamento da questão da erradicação do trabalho infantil e do trabalho forçado;

- Elaborar política de responsabilidade ambiental, discutir com os fornecedores e incluir nos contratos aspectos ambientais e sociais;

- Elaborar programas de conscientização dos funcionários quanto à redução de desperdício, reutilização e reciclagem;

- Avaliar o impacto indireto das atividades na comunidade local;

- Desenvolver atividades sistemáticas de educação ambiental para os funcionários;

- Elaborar estudos de redução do consumo de energia, água, produtos tóxicos e matérias-primas;

- Manter destinação adequada para cada resíduo, bem como o tratamento adequado dos rejeitos e resíduos;

- Criar indicadores de eficiência ambiental;

- Criar política e disponibilizar canal de informação sobre produtos com consumidores e clientes, e treinar equipe de atendimento ao cliente/consumidor;

- Registrar e comunicar internamente as reclamações dos consumidores;

- Monitorar as redes sociais, averiguando o que dizem sobre os produtos da empresa;

- Utilizar o Twitter para informação aos consumidores; 
- Definir percentual do faturamento para investimento em inovação tecnológica e produtos inovadores;

- Desenvolver canal de distribuição e criar linha de produtos ecologicamente corretos;

- Analisar impacto ambiental da cadeia de valor; e

- Acompanhar indicadores do modelo construído e avaliar o grau de sustentabilidade da empresa periodicamente.

No modelo construído, verificou-se não ser possível um resultado satisfatório trabalhando somente os critérios de maior representatividade no valor global devido à diferença das taxas ser relativamente pequena entre os critérios. 0 grande desafio será aumentar o nível em todos os critérios. A busca de melhoria será constante, bem como manter o equilíbrio entre as áreas econômica, social e ambiental.

\section{Considerações finais}

A pergunta da pesquisa teve sua resposta com a apresentação do desenvolvimento do modelo no estudo de caso, onde foi identificado um conjunto de indicadores que caracteriza a sustentabilidade da empresa. A metodologia sugerida teve a capacidade de identificar, organizar, mensurar e integrar as preocupações dos decisores nas questões de sustentabilidade.

Assim, o objetivo da pesquisa - de desenvolver um modelo de avaliação da sustentabilidade corporativa, construído com base no sistema de valores, interesses e preferências dos decisores - foi alcançado, por meio do estudo de caso, com a construção do modelo para avaliar a sustentabilidade corporativa. Os fatores relevantes que afetam o grau de alcance das propriedades das diversas áreas da empresa nos compromissos com a sustentabilidade, segundo a visão dos decisores, foram esclarecidos.

A pesquisa das abordagens de avaliação da sustentabilidade corporativa, utilizando modelos de gestão globalmente aceitos para o aperfeiçoamento dos processos e ações das empresas na busca do equilíbrio sustentável, serviu para avaliar os aspectos considerados em cada ferramenta, identificando as estratégias utilizadas para relatar o desempenho econômico, ambiental e social. No entanto, o que a empresa pesquisada buscava era um sistema de avaliação que considerasse suas particularidades e o contexto no qual está inserida.

Embora tenham aumentado o conhecimento na construção do modelo proposto, as ferramentas de sustentabilidade corporativa pesquisada necessitam ser adaptadas ao contexto da empresa. A metodologia MCDA-C permitiu a discussão e desenvolvimento de um modelo próprio, capaz de avaliar a sustentabilidade conforme os aspectos e ambiente da empresa. A
MCDA-C possibilitou aos decisores a compreensão das principais questões de sustentabilidade e ações necessárias para resolvê-las, além de proporcionar a medição de desempenho e avaliação do progresso para garantir melhorias contínuas. Os indicadores desenvolvidos refletiram a realidade empresarial, seus valores e sua cultura.

Após a análise dos resultados, os decisores visualizaram que os critérios em sustentabilidade estão interconectados e que é necessário elaborar ações conjuntas para os diversos critérios, considerando todas as áreas (econômica, social e ambiental). Entre as ações geradas a partir do modelo, destaca-se a criação de um comitê de sustentabilidade, revisão do planejamento estratégico, fortalecimento dos canais de comunicação com os stakeholders e programas de conscientização para os funcionários, fornecedores e demais envolvidos. 0 desempenho de cada um dos critérios de sustentabilidade da empresa foi avaliado e possibilitou identificar oportunidades para o aperfeiçoamento.

Uma limitação do estudo de caso foi o fato de utilizar os diretores (operações, financeiro e marketing) e gerentes (RH, compras e vendas) para representarem os interesses dos stakeholders no processo decisório. 0 que se alcançou foi meramente uma aproximação do que se pretendia, visto que na realidade os valores desses decisores representam, no fundo, os interesses da empresa frente aos diversos públicos (stakeholders).

Como recomendação para futuras pesquisas, pode-se apontar o desenvolvimento de ferramentas de tecnologia da informação que integrem os diversos softwares utilizados para a construção do modelo. Esta pesquisa poderá servir de apoio para o desenvolvimento de outros modelos de avaliação de desempenho, além de colaborar com o aumento do conhecimento sobre a metodologia multicritério de apoio à decisão - construtivista.

\section{Referências}

AZAPAGIC, A. Systems Approach to Corporate Sustainability: A General Management Framework. Process Safety and Environmental Protection, v. 81, p. 303-315, 2003.

AZAPAGIC, A.; PERDAN, S. Indicators of Sustainable Development for Industry: A General Framework. Process Safety and Environmental Protection, v. 78, n. 4, p. 243-261, 2000. http://dx.doi. org/10.1205/095758200530763

BANA E COSTA, C. A. et al. Decision Support Systems in Action: Integrated Application in a Multicriteria Decision Aid Process. European Journal of Operational Research v. 113, n. 2, p. 315-335, 1999. http://dx.doi.org/10.1016/ S0377-2217(98)00219-7

BANA E COSTA, C. A. Três convicções fundamentais na prática do apoio à decisão. Pesquisa Operacional, v. 13, n. 1, p. 9-20, 1993.

BANA E COSTA, C. A. Structuration, Construction et Exploitation dún Modèle Multicritère d'Aide à la 
Decision. 1992. Tese (Doutorado em Engenharia de Sistemas)-Universidade Técnica de Lisboa, Instituto Superior Técnico, Lisboa, 1992.

BANA E COSTA, C. A.; VANSNICK, J. C. Uma Nova Abordagem ao Problema de Construção de uma Função de Valor Cardinal: MACBETH. Investigação Operacional, v. 15, p. 15-35, 1995.

BANSAL, P. The Corporate Challenges of Sustainable Development. Academy of Management Executive, v. 16, n. 2, p. 122-131, 2002. http://dx.doi.org/10.5465/ AME.2002.7173572

BARCLAY, S. Hiview software package. London: London School of Business, 1984.

BEINAT, E. Multiattribute Value Functions for Environmental Management. Amsterdam: Timbergen Institute Research Series, 1995.

BELL, S.; MORSE, S. Sustainability Indicators. London: Measuring the Immeasurable, Earthscan, 1999.

BELLEN, H. M. V. Indicadores de sustentabilidade: uma análise comparativa. 2. ed. Rio de Janeiro: Editora FGV, 2006.

BELTON, V. Multiple criteria decision analysis - practically the only way to choose. In: HENDRY, L. C.; EGLESE, R. W. (Eds.). Operational Research Tutorial Papers: 1990. Birmingham: OR Society, 1990.

BENNETT, M.; JAMES, P. Environment-Related Performance Measurement: Current Practice and Trends. Ashridge: Ashridge Management College, 1997.

BERTO, R. M.; NAKANO, D. N. A Produção Científica nos Anais do Encontro Nacional de Engenharia de Produção: Um Levantamento de Métodos e Tipos de Pesquisa. Produção, v. 9, n. 2, p. 65-76, 2000. http://dx.doi. org/10.1590/S0103-65131999000200005

BHIMANI, A.; SOONAWALlA, K. From Conformance to Performance: the Corporate Responsibilities Continuum. Journal of Accounting and Public Policy, v. 24, n. 3, p. 165-174, 2005. http://dx.doi.org/10.1016/j. jaccpubpol.2005.03.001

BOLSA DE VALORES DE SÃO PAULO - BOVESPA. Índice de Sustentabilidade Empresarial - ISE. São Paulo: Centro de Estudo em Sustentabilidade da EAESP-FGV, 2005.

CALLENS, 1.; TYTECA, D. Towards Indicators of Sustainable Development for Firms: Concepts and Definitions. Ecological Economics, v. 28, p. 41-53, 1999. http:// dx.doi.org/10.1016/S0921-8009(98)00035-4

CAMPOS, F. M.; LEMME, C. F. Exame da Metodologia dos Índices Internacionais do Mercado Acionário e da experiência brasileira na área do investimento socialmente responsável. In: ENCONTRO NACIONAL SOBRE GESTÃO EMPRESARIAL E MEIO AMBIENTE - ENGEMA, 9., 2007, Curitiba. Anais... Curitiba, 2007. Disponível em: <http:// engema.up.edu.br/arquivos/engema/pdf/PAP0090.pdf>. Acesso em: 14 abr. 2011.

CAMPOS, L. M. S.; MELO, D. A.; MEURER, S. A. A importância dos indicadores de desempenho ambiental nos sistemas de gestão ambiental (SGA). In: ENCONTRO NACIONAL SOBRE GESTÃO EMPRESARIAL E MEIO AMBIENTE - ENGEMA, 9., 2007, Curitiba. Anais... Curitiba, 2007.

COOPER, D. R.; SCHINDLER, P. S. Métodos de Pesquisa em Administração. 7. ed. Porto Alegre: Bookman, 2003.

CROOM, S. Topic lssues and Methodological Concerns for Operations Management Research. In: EDEN DOCTORAL SEMINAR ON RESEARCH METHODOLOGY IN
OPERATIONS MANAGEMENT, 2005, Brussels, Belgium. Proceedings... Brussels, 2005.

DOW JONES SUSTAINABILITY INDEXES - DJSI. Dow Jones Sustainability World Indexes Guide. versão 9.1.DJSI, 2008. Disponível em: <http://www.sustainability-index.com>. Acesso em: 14 abr. 2011.

DUTRA, A. Metodologia para avaliar e aperfeiçoar $o$ Desempenho Organizacional: Incorporando a Dimensão Integrativa à MCDA ConstrutivistaSistêmico-Sinergética. 2003. Tese (Doutorado em Engenharia da Produção)-Universidade Federal de Santa Catarina, Florianópolis, 2003.

DYLLICK, T.; HOCKERTS, K. Beyond the business case for corporate sustainability. Business Strategy and the Environment, v. 11, p. 130-141, 2002. http://dx.doi. org/10.1002/bse.323

EDEN, C.; JONES, S.; SIMS, D. Messing About in Problems. Oxford: Pergamon, 1983.

ELKINGTON, J. Cannibals with Forks: the Triple Bottom line of 21st Century Business. Capstone: Oxford, 1997.

ENSSLIN, L.; CAMPOS, M. L. A prática do aprendizado organizacional. Revista Gestão Industrial, v. 2, n. 1, p. 59-69, 2006. Disponível em: <http://www.pg.cefetpr. br>. Acesso em: 05 ago. 2009.

ENSSLIN, L.; DUTRA, A.; ENSSLIN, S. R. MCDA: A Constructivist Approach to the Management of human Resources at a Governmental Agency. International Transactions in Operational Research-ITORS, v. 7, p. 79-100, 2000.

ENSSLIN, L.; MONTIBELLER, G. N. From Cognitive Maps to Multicriteria Models: Toward a Formal Procedure for the Transition. In: INFORMS CONFERENCE - Israel '98, 1998, Tel Aviv, lsrael. Proceedings... Tel Aviv, 1998.

ENSSLIN, L.; MONTIBELLER, G. N.; NORONHA, S. M. Apoio à decisão: metodologias para estruturação de problemas e avaliação multicritério de alternativas. Florianópolis: Insular, 2001.

ENSSLIN, L.; NORONHA, S. M. Uma visão geral do software HIVIEW. Laboratório de Metodologias Multicritério em Apoio à Decisão, Universidade Federal de Santa Catarina, 1997.

ENSSLIN,S.R. Incorporaçãodaperspectivasistêmico-sinergética na metodologia MCDA construtivista: uma ilustração de implementação. 2002. Tese (Doutorado em Engenharia de Produção)-Universidade Federal de Santa Catarina, Florianópolis, 2002.

EPSTEIN, M. J.; ROY, M. J. Improving Sustainability Performance: Specifying, Implementing and Measuring Key Principles. Journal of General Management, v. 29, n. 1, p. 15-31, 2003.

FINANCIAL TIMES STOCK EXCHANG - FTSE. Ground Rules for the Management of the FTSE4Good Index Series. versão 1.3. Londres, 2005. Disponível em: <http://www. ftse.com/ftse4good>. Acesso em: 14 abr. 2011.

FORZA, C. Survey Research in Operations Management: a Process-based Perspective. International Journal of Operations \& Production Management, v. 22 , n. 2 , p. 152-194, 2002. http://dx.doi. org/10.1108/01443570210414310

GIFFHORN, E. Construção de modelo de avaliação de desempenho de empresas terceirizadas com a utilização da metodologia MCDA-C. 2007. Dissertação (Mestrado em Engenharia de Produção) - Universidade Federal de Santa Catarina, Florianópolis, 2007.

GLADWIN, T.; KENNELY, J.; KRAUSE T. S. Shifting paradigms for sustainable development: implications 
for management theory and research. Academy of Management Review, v. 20, n. 4, p. 874-907, 1995.

GLOBAL REPORTING INITIATIVE - GRI. Diretrizes para Relatório de Sustentabilidade. Versão em português. São Paulo, 2006. Disponível em: <http://www. globalreporting.org>. Acesso em: 14 abr. 2011.

GODOY, A. S. Introdução à pesquisa qualitativa e suas possibilidades. RAE - Revista de Administração de Empresas, v. 35, n. 3, 1995.

HANLEY, N. Macroeconomic Measures of 'Sustainability'. Journal of Economic Surveys, v. 14, p. 1- 30, 2000. http://dx.doi.org/10.1111/1467-6419.00102

INSTITUTO ETHOS. Guia para Elaboração de Balanço Social e Relatório de Sustentabilidade. São Paulo: Instituto Ethos, 2008. Disponivel em: <http://www.ethos.org.br>. Acesso em: 14 abr. 2011.

KEEBLE, J. J.; TOPIOL, S.; BERKELEY, S. Using Indicators to Measure Sustainability Performance at a Corporate and Project Level. Journal of Business Ethics, v. 44, n. 2, p. 149-158, 2002. http://dx.doi. org/10.1023/A:1023343614973

KEENEY, R. L. Value-Focused Thinking: A Path to Creative Decision-making. Cambridge: Harvard University Press, 1992.

KEENEY, R. L.; RAIFFA, H. Decision with Multiple Objectives, Preferences and Value Tradeoffs. Cambridge: Cambridge University Press, 1993.

LANDRY, M. A note of the concept of problem. European Group for Organizations Studies, v. 16, p. 2315-2343, 1995.

LOUETTE, A. Gestão do Conhecimento - Compêndio para Sustentabilidade: Ferramentas de Gestão de Responsabilidade Socioambiental. Antakarana Cultura Arte Ciência / Willis Harnan House. São Paulo, 2007.

MATTAR, F. N. Pesquisa de Marketing: Metodologia e Planejamento. São Paulo: Atlas, 1996.

MAZON, R.; MONZONI, M.; RIBEIRO, G. Combate à Biopirataria precisa preservar bons pesquisadores. Revista Brasileira de Desenvolvimento Sustentável - Brazil Forever, v. 20, n. 4, p. 26-28, 2005.

MIGUEL, P. A. C. Estudo de caso na engenharia de produção: estruturação e recomendações para sua condução. Produção, v. 17, n. 1, p. 216-229, 2007. http://dx.doi. org/10.1590/S0103-65132007000100015

MILLER, D. W. Estrutura das decisões humanas. Rio de Janeiro: Fundação Getúlio Vargas, 1970.
NASCIMENTO, L. F.; LEMOS, A. D. C.; MELLO, M. C. A. Gestão Sociambiental Estratégica. Porto Alegre: Bookman, 2008.

NEELY, A. Performance Measurement System Design: A Process-based Approach. Cambridge: Manufacturing Engineering Group, University of Cambridge, 1993.

OSBORN, A. F. Applied Imagination. 3. ed. Buffalo: Creative Education Foundation, 1993.

ROBERTS, F. S. Measurement Theory. In: ROTA, G. C. (Ed.) Encyclopedia of Mathematics and its Applications. London: Addison Wesley Publishing Company, 1979. v. 7.

ROY, B. Méthodologie Multicritère d'Aide à la Décision. Paris: Economica, 1985.

ROY, B. Multicriteria Methodology for Decision Aiding. Dordrecht: Kluwer Academic Publishers, 1996.

ROY, B. On operational research and decision aid. European Journal of Operational Research, v. 73, n. 1, p. 23-26, 1994. http://dx.doi.org/10.1016/0377-2217(94)90136-8

SCHAEFER, N. R. Modelo para Analisar o Perfil Profissional de um Gerente. 2005. Dissertação (Mestrado em Engenharia de Produção)-Universidade Federal de Santa Catarina, Florianópolis, 2005.

SCHALTEGGER, S.; BURRITT, R.; PETERSEN, H. An Introduction to Corporate Environmental Management. Sheffield: Striving for Sustainability, 2003.

SCHALTEGGER, S.; WAGNER, M. Integrative management of sustainability performance, measurement and reporting. International Journal of Accounting, Auditing and Performance Evaluation, v. 3, n. 1, p. 1-19, 2006.

SIMON, H. A. Comportamento administrativo: estudo dos processos decisórios nas organizações administrativas. Rio de Janeiro: Editora da Fundação Getúlio Vargas, 1979.

SOUZA, R. Case Research in Operations Management. In: EDEN DOCTORAL SEMINAR ON RESEARCH METHODOLOGY IN OPERATIONS MANAGEMENT, 2005, Brussels, Belgium. Proceedings... Brussels, 2005.

TRIGUEIRO, A. (Org.). Meio ambiente no século 21: 21 especialistas falam da questão ambiental nas suas áreas de conhecimento. 5. ed. Campinas: Armazém do Ipê, 2008.

WEHRMEYER, W.; TYTECA, D. Measuring Environmental Performance For Industry: From Legitimacy To Sustainability? International Journal of Sustainable Development and World Ecology, v. 5, p. 111-124, 1998. http://dx.doi.org/10.1080/13504509809469975

\section{Development of a model for corporate sustainability assesment Abstract}

This paper introduces the development of an assessment model for corporate sustainability, which was built based on the system of values, concerns and preferences of decision makers from a textile factory. The Multi-criteria for Decision Aid - Constructivist (MCDA-C) methodology was used, enabling to identify a set of performance indicators that features the company's sustainability and to measure the degree of properties range considered by the organization about its commitment to sustainability. The model allowed decision makers to understand the key sustainability issues and actions needed to resolve them, and provide performance measurement and evaluation of progress toward continuous improvement. Among the actions generated by the model, there are the creation of a committee of sustainability, strategic planning review, strengthening of communication channels and awareness programs for employees, suppliers and others involved.

Keywords

Decision aid. Performance assessment. MCDA-C. Corporate sustainability. 